\title{
A simple method for computing sprint acceleration kinetics from running velocity data: replication study with improved design
}

\author{
Jean-Benoit Morin ${ }^{a, d}$, Pierre Samozino ${ }^{b}$, Munenori Murata ${ }^{c}$, Matt R Cross ${ }^{b, d}$, Ryu Nagahara ${ }^{c}$ \\ a Université Côte d'Azur, LAMHESS, Nice, France. \\ buniv Savoie Mont Blanc, Laboratoire Interuniversitaire de Biologie de la Motricité, EA 7424, F-73000 Chambéry, France \\ 'National Institute of Fitness and Sports in Kanoya, Kanoya, Japan \\ ${ }^{\mathrm{d}}$ Sports Performance Research Institute New Zealand (SPRINZ), Auckland University of Technology, Auckland, New Zealand \\ Corresponding author: Pr Jean-Benoit Morin, Faculty of Sport Science \\ 261 Boulevard du Mercantour, 06205 NICE, France \\ jean-benoit.morin@univ-cotedazur.fr
}

\begin{abstract}
Measuring the ground reaction forces (GRF) underlying sprint acceleration is important to understanding the performance of such a common task. Until recently direct measurements of GRF during sprinting were limited to a few steps per trial, but a simple method (SM) was developed to estimate GRF across an entire acceleration. The SM utilizes displacement- or velocity-time data and basic computations applied to the runner's center of mass and was validated against compiled force plate (FP) measurements; however, this validation used multiple-trials to generate a single acceleration profile, and consequently fatigue and error may have introduced noise into the analyses. In this study, we replicated the original validation by comparing the main sprint kinetics and force-velocity-power variables (e.g. GRF and its horizontal and vertical components, mechanical power output, ratio of horizontal component to resultant GRF) between synchronized FP data from a single sprinting acceleration and SM data derived from running velocity measured with a $100 \mathrm{~Hz}$ laser. These analyses were made possible thanks to a newly developed 50-m FP system providing seamless GRF data during a single sprint acceleration. Sixteen trained male sprinters performed two all-out 60-m sprints. We observed good agreement between the two methods for kinetic variables (e.g. grand average bias of $4.71 \%$, range $0.696 \pm 0.540-8.26 \pm 5.51 \%$ ), and high inter-trial reliability (grand average standard error of measurement of $2.50 \%$ for FP and $2.36 \%$ for the SM). This replication study clearly shows that when implemented correctly, this method accurately estimates sprint acceleration kinetics.
\end{abstract}

KEYWORDS: force; power; running; force platform; sports performance 


\section{Introduction}

Sprint running acceleration is a major component of performance in many sports, and ground reaction forces (GRFs) acting on the runner's body basically explain the overall linear sprint acceleration motion. Thus, quantifying the main sprint kinetics is key for a better understanding of human sprint acceleration motion and performance (Cavagna et al., 1971; Rabita et al., 2015). In parallel, measuring kinetics (e.g. GRF or external mechanical power output) during this task has historically been an experimental challenge (Cross et al., 2017a; Furusawa et al., 1927). Although displacement- and velocity-time outputs have been quantified for decades (Best and Partridge, 1928; Volkov and Lapin, 1979), the first publications on overground sprint acceleration kinetics are comparatively recent (e.g. Cavagna et al., 1971; Rabita et al., 2015). The reference technology for measuring GRF in these seminal studies are force platforms installed into the surface over which the athlete is running; in this manner, direct and high-fidelity data are gathered in a highly specific and non-invasive manner. One major limitation of these studies is that datasets were restricted by the number of force platforms (FP) arranged in series (typically 4 to $8 \mathrm{~m}$ in total). As such, studying a complete sprint acceleration (20 to $60 \mathrm{~m}$ depending on the athlete's characteristics) required data to be aggregated from multiple sprints to form a compiled "single" acceleration profile (Rabita et al., 2015). Recently, Samozino et al. (2016) proposed a simple method based on a macroscopic model applying the fundamental laws of motion to the runner's center of mass. Using this method, mechanical outputs determined from simple kinematic (spatiotemporal) data showed good to very good agreement to those assessed from a reference FP system. Because it is based on basic inputs (body mass, height, and displacement- or velocity-time data), this method has been widely used in sports practice and research (e.g. Mendiguchia et al., 2016; Pantoja et al., 2016; Jimenez-Reyes et al., 2018; Cross et al., 2015). Despite the clear and strong results from the initial validation study, the validity of this approach has only been tested in the context of a multiple-trial design. Moreover, while the methodology in this original validation was sound, the multiple trial approach is subject to some unavoidable variability that may have affected the results (Samozino et al., 2016). As such, some may argue that the outputs from the simple method are somewhat invalid due to not being compared to force plate data collected concomitantly over a single sprint trial. A unique system of track-embedded FP was recently developed allowing $52 \mathrm{~m}$ of continuous and synchronized force measurements, owing to its "series" construction within an indoor 110-m sprint track (Nagahara et al., 2017a; Colyer et al., 2018); thus, the validity of Samozino et al.'s simple method can be tested using such a system by comparing the two data streams collected simultaneously during a single sprint acceleration. The aim of this study was to replicate Samozino et al.'s test of the criterion validity and estimation errors of the simple computation method (Samozino et al., 2016) against reference FP data, this time with direct, single sprint comparisons.

\section{Method}

2.1. Participants, procedures and equipment

Sixteen male trained sprinters (mean \pm SD age of $20.4 \pm 1.5 \mathrm{yr}$; mass $65.0 \pm 3.5 \mathrm{~kg}$; height $1.72 \pm 0.03 \mathrm{~m}$; and $100-\mathrm{m}$ personal best time $11.2 \pm 0.4 \mathrm{~s}$ ) gave their informed, written consent to participate in this study, that was approved by the research ethics committee of the National Institute of Fitness and Sports in Kanoya. After a complete and appropriate warm-up, athletes performed two all-out 60-m sprints (separated by $>10 \mathrm{~min}$ ) on an indoor running track, using their preferred crouched block-start position and their own sprinting spiked shoes. A series of fifty-four platforms (1000 Hz; TF-90100, TF-3055, TF-32120; Tec Gihan, Uji, Japan, see here for details: http://www.tecgihan.co.jp/en/products/forceplate/) were embedded into the indoor track, and connected to a single computer to measure GRF through $52 \mathrm{~m}$ from $1.5 \mathrm{~m}$ behind the starting line to the 50.5 -m mark (for more details on the FP procedures and equipment, please see Colyer et al. 2018, Nagahara et al. 2017a). During each trial, athletes' running velocity was also computed from the position-time data (see detailed procedures in Nagahara et al. 2016) measured with a laser system ( $100 \mathrm{~Hz}$; LDM301, Jenoptik, Jena, Germany) set at $1.0 \mathrm{~m}$ above the ground 
and aimed at the lower back of the runners. The two systems were synchronized via electric starting gun that provided a starting signal for participants.

\subsubsection{Simple method}

The complete description of the simple method used in this study can be read elsewhere (Samozino et al., 2016). Briefly, in this macroscopic method based on the motion of the body center of mass, running velocity over time measured by laser during the acceleration (starting at a $0.1 \mathrm{~m} / \mathrm{s}$ threshold) was fitted with an exponential function using the least-square regression method (di Prampero et al., 2015; Furusawa et al., 1927; Volkov and Lapin, 1979; Samozino et al., 2016), with a time adjustment to ensure the actual start of the computation at $t=0 \mathrm{~s}$, in case of delay between the time trigger and the actual increase in velocity (Samozino, 2018):

$v_{H}(t)=v_{H \max } \cdot\left(1-e^{-(t-d) / \tau}\right)$

with $v_{H \max }$ the maximal velocity (in $\mathrm{m} / \mathrm{s}$ ) reached at the end of the acceleration, the acceleration time constant (in s) and $d$ the time delay $(0.029 \pm 0.042$ s on average in the present study) (Fig. 1).

Then, the acceleration of the runner in the horizontal direction was computed by derivating $v_{H}$ over time:

$a_{H}(t)=\left(\frac{v_{H \max }}{\tau}\right) \cdot e^{-\frac{t}{\tau}}$

Applying the fundamental laws of dynamics in the horizontal direction, the net horizontal antero-posterior ground reaction force $\left(F_{H}\right)$ applied to the body center of mass $(C M)$ was modelled over time as:

$F_{H}(t)=m a_{H}(t)+F_{\text {aero }}(t)$

with $m$ the athlete's body mass and $F_{\text {aero }}$ the aerodynamic drag force, estimated from the athlete's body mass and height, ambient air temperature and barometric pressure, and running velocity (see Arsac and Locatelli, 2002). This aerodynamic drag necessary to overcome during sprinting is proportional to the square of the velocity of air relative to the runner:

$F_{\text {aero }}(t)=k \cdot\left(v_{H}(t)-v_{W}\right)^{2}$

where $v_{w}$ is the wind velocity (if any) and $k$ is the runner's aerodynamic friction coefficient, estimated as proposed by Arsac and Locatelli (2002) from values of air density $\left(\boldsymbol{\rho}\right.$, in $\left.\mathrm{kg} / \mathrm{m}^{3}\right)$, frontal area of the runner $\left(A f\right.$, in $\left.\mathrm{m}^{2}\right)$, and drag coefficient $(C d=0.9)$ :

$k=0.5 \cdot \rho \cdot A f \cdot C d$

$\rho=\rho_{0} \cdot \frac{P b}{760} \cdot \frac{273}{273+T^{\circ}}$

$A f=\left(0.2025 \cdot h^{0.725} \cdot m^{0.425}\right) \cdot 0.266$

where $\boldsymbol{\rho}_{0}=1.293 \mathrm{~kg} / \mathrm{m}$ is the $\boldsymbol{\rho}$ at 760 Torr and $273{ }^{\circ} \mathrm{K}, \mathrm{Pb}$ is the barometric pressure (in Torr), $T^{\circ}$ is the air temperature (in ${ }^{\circ} \mathrm{C}$ ), and $h$ is the runner's stature (in $\mathrm{m}$ ). Although this method overestimates the frontal area during early acceleration, the resultant error is likely negligible due to associated low CM velocity. 
Similar facilities meant ambient conditions were close between the current study and the original one (Samozino et al., 2016). The mean net horizontal antero-posterior power output applied to the body center of mass $\left(P_{H}\right)$ was modelled at each instant as the product of $F_{H}$ and $v_{H}$ (Fig. 2). The ratio of $F_{H}$ to the resultant GRF $\left(F_{R E S}\right)$ was computed over time according to Morin et al. (Morin et al., 2011) and expressed in \%:

$R F=\frac{F_{H}}{F_{R E S}}=\frac{F_{H}}{\sqrt{F_{H}^{2}+F_{V}^{2}}}$

with $F_{V}$ the vertical component of the GRF, considered as equal to body weight over time (di Prampero et al., 2015; Samozino et al., 2016). RF is an indicator of the overall orientation of the GRF vector during the step, which represents the ability to apply the force they generate onto the ground in an "effective", i.e. horizontally-oriented manner (Morin et al., 2011; Rabita et al., 2015).

\subsubsection{Force platform reference method}

As fully detailed elsewhere (Nagahara et al., 2017a; Colyer et al., 2018), the GRF data were firstly filtered using a fourth-order low-pass Butterworth filter with a 70-Hz cut-off frequency derived through residual analysis. Raw $F_{H}(t)$ and $F_{V}(t)$ signals (Fig. 1) were then averaged for each step (time ranging from one foot contact to the contact of the contralateral foot), as determined with thresholds of $F_{V}=20 \mathrm{~N}$. Horizontal velocity was calculated for each step using the impulse-momentum relationship (Fig. 1).

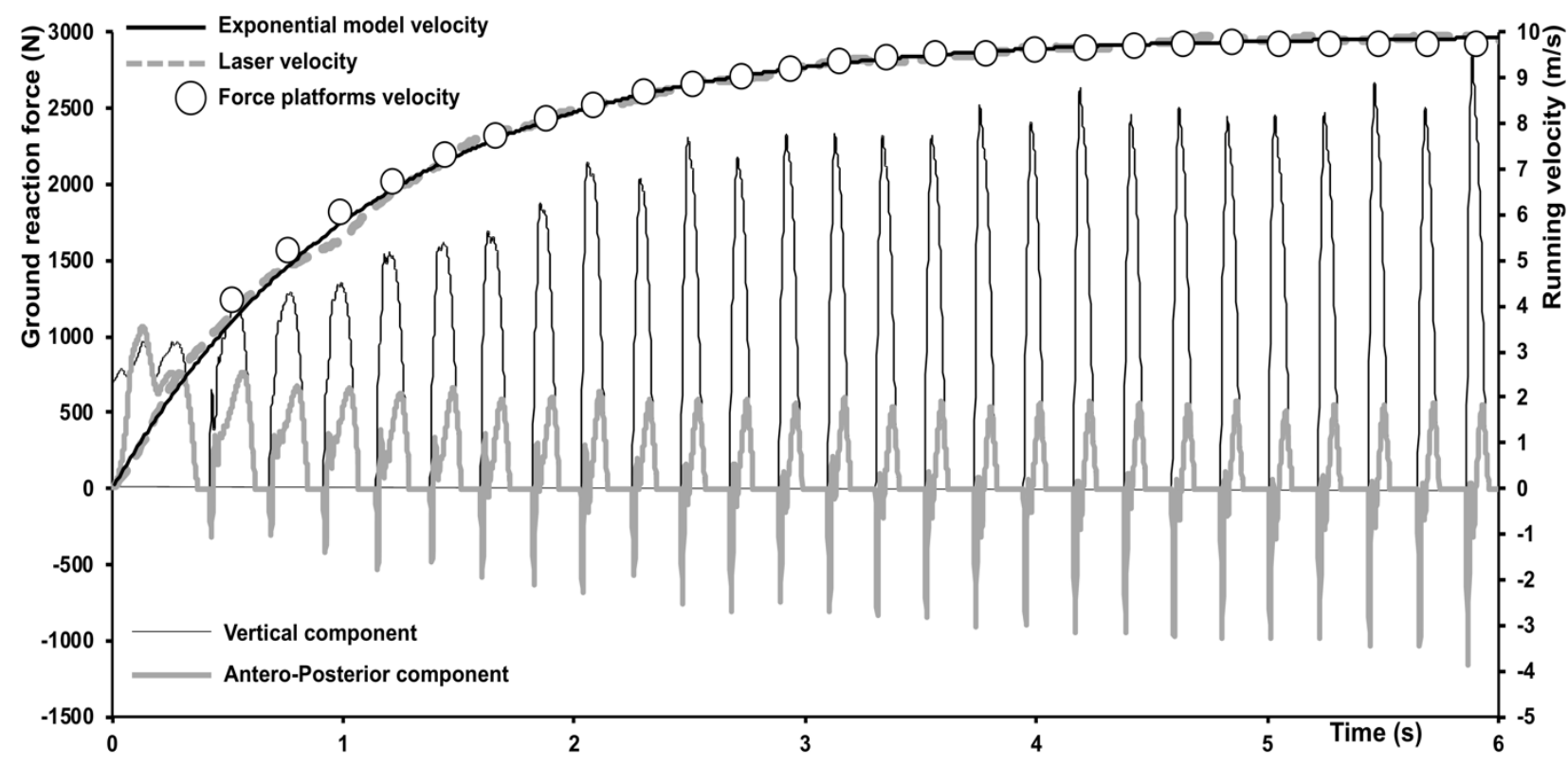

Fig. 1. Typical example of raw data of running velocity, vertical and antero-posterior components of the ground reaction force during the first 6 seconds of a 60-m sprint acceleration. Circles indicate step-averaged values of running velocity, over the corresponding step, after the starting-block push-off. The running velocity traces obtained from the laser system and the associated exponential fitting are almost overlapping. 


\subsection{Common data analysis}

From modelled (simple method) and step-averaged values of $F_{H}(t), v_{H}(t)$ and $P_{H}(t)$ data, we computed the linear force-velocity relationship, second degree polynomial power-velocity relationship and linear $R F$-velocity relationship (Cross et al., 2017a; Rabita et al., 2015, Morin et al., 2011) (Fig. 3). Then, from these relationships, the following variables were computed: the theoretical maximal horizontal force output $F_{H 0}$ and running velocity $v_{H 0}$ (calculated as the intercepts of the force-velocity relationship), the slope of the force-velocity relationship $\left(S_{F v}\right)$, the maximal power output:

$P \max =\frac{F_{H 0}}{2} \cdot \frac{v_{H 0}}{2}$

and the decrease in the ratio of force $\left(D_{R F}\right)$ index, computed as the slope of the linear decrease in $R F$ with increasing

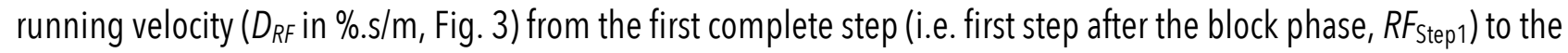
step at maximal velocity (Morin et al., 2011).

\subsection{Statistics}

All data are presented as mean \pm standard deviation (SD). The quality of the exponential fitting (Eq. 1) of laser and FP running velocity data, and the adjustments of the force-, power- and RF-velocity relationships to their respective linear or second-degree polynomial models were assessed with $R^{2}$ values for both laser and FP data (Fig.1 and Fig.3). For each subject, the standard errors of estimate (SEE) for $F_{H}, F_{V}, F_{R E S}$ and $R F$ were computed between averaged FP values for each step and values estimated from the simple method, for corresponding step-averaged $v_{H}(t)$ values:

$S E E=\sqrt{\frac{\sum\left(F_{F P}-F_{\text {Simplemethod }}\right)^{2}}{N_{\text {steps }}-2}}$

The main kinetic variables of the force-velocity-power relationships obtained with both methods were then compared using bias (mean differences between both methods, systematic bias), random errors (1.96 x standard deviation of the differences between both methods) and limits of agreement (bias + random errors, Bland and Altman, 1986). In case of significant correlation between individual values and residuals, data were log-transformed before further analyses (Atkinson and Nevill, 1998). Finally, the inter-trial reliability for each variable was quantified using the coefficient of variation (CV in \%), the change in the mean, and the standard error of measurement (SEM, expressed in percentage of mean values) between the two trials (Hopkins, 2000).

\section{Results}

As seen in the typical example shown in Fig. 1, the exponential model provided almost perfect fitting of $v_{H}(t)$ data, for both laser $\left(R^{2}>0.996\right)$ and $F P\left(R^{2}>0.999\right)$ measurements. As shown in Fig. 3, force- and $R F$-velocity relationships obtained with the FP were very well fitted by linear regressions (mean $R^{2}$ of $0.955 \pm 0.022$ for force-velocity and $0.975 \pm 0.013$ for $R F$-velocity) and power-velocity relationships by second degree polynomial regressions (mean $\mathrm{R}^{2}$ of $0.920 \pm 0.033$ ).

The FP values of $F_{H}, F_{V}, F_{R E S}$ and $R F$ at each step were well fitted by the simple method proposed, as shown by the SEE of $25.8 \pm 6.3 \mathrm{~N}, 37.6 \pm 13.7 \mathrm{~N}, 45.3 \pm 11.5 \mathrm{~N}$ and $2.23 \pm 0.41 \%$, respectively. This is illustrated in the typical example shown in Fig. 2. 


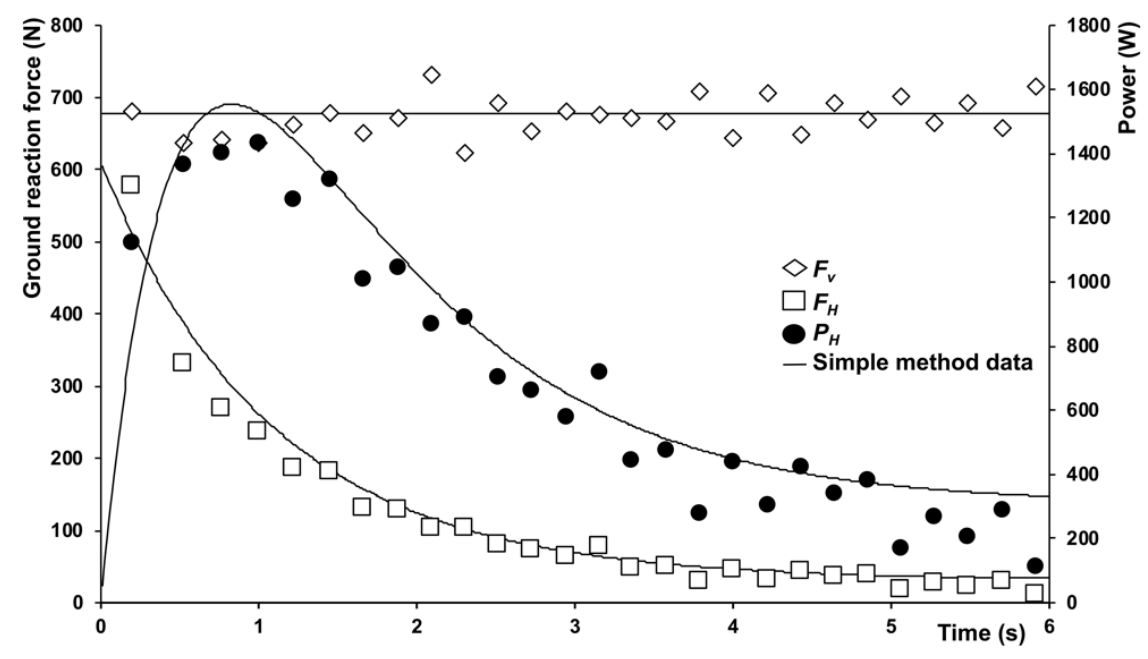

Fig. 2. Typical example of step-averaged values of vertical and antero-posterior components of the ground reaction force, and associated power output over the first $6 \mathrm{~s}$ of a 60 -m sprint including starting-blocks push-off. The simple method data for this trial appear as black traces for each variable. Data from the same individual and trial as in Fig. 1.

All other comparison results between the reference FP data and the simple method proposed are shown in Table 1.

Table 1. Mean \pm SD of the main mechanical variables and comparison between the force platform (reference) and simple method data.

\begin{tabular}{lcccccc}
\hline & $\begin{array}{c}\text { Force } \\
\text { platform } \\
\text { method }\end{array}$ & $\begin{array}{c}\text { Simple } \\
\text { method }\end{array}$ & $\begin{array}{c}\text { Ratio of simple } \\
\text { method / } \\
\text { reference } \\
\text { method (after } \\
\text { log transform) }\end{array}$ & $\begin{array}{c}\text { Systematic } \\
\text { error (Mean } \\
\text { of inter- } \\
\text { method } \\
\text { differences) }\end{array}$ & $\begin{array}{c}\text { Random } \\
\text { error } \\
\text { (1.96xSD of } \\
\text { inter-method } \\
\text { differences) }\end{array}$ & $\begin{array}{c}\text { 95\% } \\
\text { agreement } \\
\text { limits }\end{array}$ \\
\hline $\boldsymbol{v}_{\max }(\mathrm{m} / \mathrm{s})$ & $9.58 \pm 1.24$ & $9.55 \pm 0.34$ & 0.997 & -0.033 & 0.153 & $(-0.186 ; 0.120)$ \\
$\boldsymbol{T a u}(\mathrm{s})$ & $1.19 \pm 0.09$ & $1.15 \pm 0.07$ & 0.929 & -0.088 & 0.157 & $(-0.251 ; 0.063)$ \\
$\boldsymbol{F}_{\text {HO }}(\mathrm{N} / \mathrm{kg})$ & $8.59 \pm 0.57$ & $8.15 \pm 0.48$ & 0.948 & -0.444 & 0.745 & $(-1.21 ; 0.277)$ \\
$\boldsymbol{V}_{\text {HO }}(\mathrm{m} / \mathrm{s})$ & $9.89 \pm 0.37$ & $9.98 \pm 0.38$ & 1.01 & 0.091 & 0.198 & $(-0.107 ; 0.289)$ \\
$\boldsymbol{S}_{\mathrm{Fv}}(\mathrm{N} / \mathrm{kg} / \mathrm{s} / \mathrm{m})$ & $-0.870 \pm 0.066$ & $-0.818 \pm 0.052$ & 0.941 & 0.051 & 0.079 & $(-0.025 ; 0.133)$ \\
$\boldsymbol{P}_{\max }(\mathrm{W} / \mathrm{kg})$ & $21.2 \pm 1.7$ & $20.4 \pm 1.5$ & 0.959 & -0.877 & 1.84 & $(-2.76 ; 0.925)$ \\
$\boldsymbol{R F}_{\mathrm{Step}}(\%)$ & $44.2 \pm 2.9$ & $43.4 \pm 2.0$ & 0.981 & -0.825 & 5.92 & $(-6.76 ; 5.08)$ \\
$\boldsymbol{D}_{\boldsymbol{R F}}(\% / \mathrm{s} / \mathrm{m})$ & $-7.33 \pm 0.57$ & $-7.20 \pm 0.45$ & 0.982 & 0.130 & 0.694 & $(-0.561 ; 0.827)$ \\
\hline
\end{tabular}
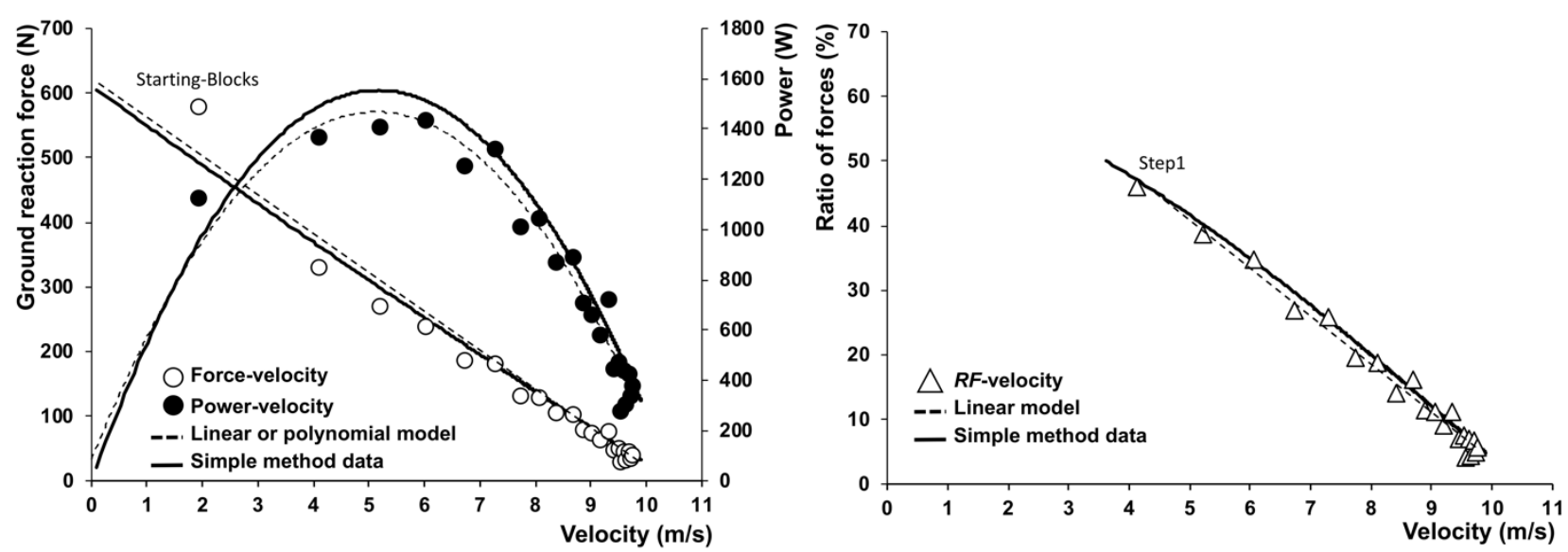

Fig. 3. Left: typical example of force- and power-velocity relationships computed from step-averaged values (force platform circles and dashed traces) and using the simple method (black traces). Right: ratio of force as a function of running velocity for the same sprint trial. Data from the same individual and trial as in Fig. 1 and Fig. 2. 
Finally, the inter-trial variability was low, and very close between the two methods (Table 2). For example, average coefficients of variation between the two trials (for both $\mathrm{FP}$ and the simple method) for $V_{H \max }, F_{H O}, V_{H O}$ and $P_{\max }$ were all $<3.56 \pm 2.00 \%$.

Table 2. Inter-trial reliability variables for the force platform (reference) and simple method data.

$$
\text { Coefficient of variation Change in the mean Standard error of }
$$

(\%)

(\%)

measurement (\%)

\begin{tabular}{lcccccc}
\hline & $\begin{array}{c}\text { Force } \\
\text { platform } \\
\text { method }\end{array}$ & $\begin{array}{c}\text { Simple } \\
\text { method }\end{array}$ & $\begin{array}{c}\text { Force } \\
\text { platform } \\
\text { method }\end{array}$ & $\begin{array}{c}\text { Simple } \\
\text { method }\end{array}$ & $\begin{array}{c}\text { Force } \\
\text { platform } \\
\text { method }\end{array}$ & $\begin{array}{c}\text { Simple } \\
\text { method }\end{array}$ \\
\hline $\boldsymbol{V}_{\max }(\mathrm{m} / \mathrm{s})$ & 0.674 & 0.481 & 0.563 & 0.303 & 0.864 & 0.667 \\
$\boldsymbol{T a u}(\mathrm{s})$ & 2.24 & 3.41 & 0.944 & -1.59 & 2.82 & 4.00 \\
$\boldsymbol{F}_{\text {HO }}(\mathrm{N} / \mathrm{kg})$ & 2.03 & 3.51 & -0.217 & 1.94 & 2.53 & 3.81 \\
$\boldsymbol{V}_{\boldsymbol{H O}}(\mathrm{m} / \mathrm{s})$ & 0.473 & 0.555 & 0.271 & 0.319 & 0.535 & 0.757 \\
$\boldsymbol{S}_{\mathrm{Fv}}(\mathrm{N} / \mathrm{kg} / \mathrm{s} / \mathrm{m})$ & 2.00 & 3.56 & -0.478 & 1.306 & -2.55 & -3.95 \\
$\boldsymbol{P}_{\text {max }}(\mathrm{W} / \mathrm{kg})$ & 2.11 & 3.07 & 0.032 & 1.956 & 2.61 & 3.30 \\
$\boldsymbol{R}_{\text {Step } 1}(\%)$ & 4.26 & 1.31 & 1.379 & 0.349 & 5.64 & 1.63 \\
$\boldsymbol{D}_{\boldsymbol{R F}}(\% / \mathrm{s} / \mathrm{m})$ & 2.25 & 3.23 & 0.278 & 0.916 & -2.87 & -3.72 \\
\hline
\end{tabular}

\section{Discussion}

Overall, this replication study with improved experimental design (i.e. single sprint direct data comparison versus indirect multiple-sprint approach) clearly confirms the results obtained by Samozino et al. (2016): (i) velocity-time data during a sprint acceleration (measured with FP or laser devices) was almost perfectly described by a simple exponential model (Eq. 1); (ii) the main macroscopic force-velocity-power outputs computed using simple laws of motion applied to the runner's CM (Samozino et al., 2016) exhibited good agreement with reference force-plate measurements (grand average bias of $4.71 \%$, Table 1), and; (iii) the inter-trial variability in sprint kinetics was low in the trained population studied, and very close between the two methods compared (Table 2).

In this population of trained male sprinters, sprint kinetics data were similar to those obtained in previous studies using either FP (Rabita et al., 2015; Nagahara et al., 2017a; Colyer et al., 2018) or Samozino et al.'s simple method (e.g. Slawinski et al., 2017; Cross et al., 2017b; Jimenez-Reyes et al., 2018). It is also interesting to note that the low inter-trial variability observed here was very close between the two methods compared (Table 2), and comparable to that reported by Haugen et al. (2018) in a similar population, using the simple method approach. Simperingham et al. (2017) reported clearly higher values for individuals of a lower level of performance, which might indicate that the inter-trial variability in sprint kinetics is more related to the characteristics of the individuals tested, rather than the measurement and computation method used.

The almost perfect fit of the velocity-time data by the exponential model (Eq. 1) confirms previous studies (di Prampero et al., 2015; Furusawa et al., 1927; Volkov and Lapin, 1979; Samozino et al., 2016). In addition, all subsequent computations of sprint kinetics using Samozino et al.'s method are similar and not device-dependent. Thus, it appears that other types of displacement or velocity-time devices than the laser may be used, provided the almost perfect exponential fitting is verified. For example, previous studies used radar (Cross et al., 2015), photocells (Samozino et al., 2016; Romero-Franco et al., 2017), linear encoders (Cross et al., 2018), or high-speed video (Romero-Franco et al., 2017) after verification of the high-quality of fitting (correlation coefficient $>0.99$ ). In addition, this almost perfect fit was observed in loaded sprint conditions (Pantoja et al., 2018; Cross et al. 2017b; Cross et al. 
2018) and for various levels of sprint performance and athletes' age and sex (Pantoja et al., 2016; Slawinski et al., 2017; Nagahara et al., 2017b), which tends to support its general validity to varying athlete's characteristics/levels and with external resistance. However, if high-quality exponential fitting of the data is not verified preceding analysis, any subsequent computations might lead to inaccurate data and conclusions (e.g. GPS (Nagahara et al., 2017b) and inertial unit devices data (Morin et al., 2018)). As for the inter-trial reliability, the overall accuracy of the simple method highly depends on the initial accuracy of the position- or velocity-time measurements, rather than the computations themselves which are based on the laws of motion.

One limitation of the present study is that it was performed only in trained male sprinters, using a starting-block start, and not to other types of participants (e.g. team sport players) and starting positions (e.g. 3-point start, standing start). That said, given the results presented here and the points previously discussed, we can reasonably expect that provided accurate basic inputs (body mass, height, and displacement- or velocity-time) and a verified exponential fit (very high-quality of fit of the velocity-time data with the exponential model from Eq. 1), the final outputs of the simple method would show the same level of agreement with FP data, and the same level of inter-trial reliability. Following the strong results of the initial validation study, this quasi-replication study clearly proves the validity of the method.

In conclusion, this replication study using a single sprint approach and reference force plate data confirms the initial validation study (Samozino et al., 2016): even though force plate remains the gold standard method for measuring sprint acceleration kinetics and force-velocity-power outputs, especially with access to within-step and between-limbs GRF data, the proven validity and reliability of Samozino et al.'s simple computation confirms it as a feasible alternative for those working in field conditions or without access to specialists devices. When implemented correctly, this method is a useful approach for sports and health research and practice.

\section{Conflict of interest statement}

The authors declare that they have no conflict of interest.

\section{References}

Arsac, L.M., Locatelli, E., 2002. Modeling the energetics of 100-m running by using speed curves of world champions. J. Appl. Physiol. 92, 1781-1788. https://doi.org/10.1152/japplphysiol.00754.2001

Atkinson, G., Nevill, A.M., 1998. Statistical methods for assessing measurement error (reliability) in variables relevant to sports medicine. Sports Med. 26, 217-238.

Bland, J.M., Altman, D.G., 1986. Statistical methods for assessing agreement between two methods of clinical measurement. Lancet (London, England) 1, 307-10.

Best, C.H., Partridge, R.C., 1928. The Equation of Motion of a Runner, Exerting a Maximal Effort. Proc. R. Soc. B Biol. Sci. 103, 218-225. https://doi.org/10.1098/rspb.1928.0036

Cavagna, G.A., Komarek, L., Mazzoleni, S., 1971. The mechanics of sprint running. J. Physiol. 217, 709-721. https://doi.org/10.1113/jphysiol.1971.sp009595

Colyer, S.L., Nagahara, R., Salo, A.I.T., 2018. Kinetic demands of sprinting shift across the acceleration phase: Novel analysis of entire force waveforms. Scand. J. Med. Sci. Sport. https://doi.org/10.1111/sms.13093

Cross, M.R., Lahti, J., Brown, S.R., Chedati, M., Jimenez-Reyes, P., Samozino, P., Eriksrud, O., Morin, J.-B., 2018. Training at maximal power in resisted sprinting: Optimal load determination methodology and pilot results in team sport athletes. PLoS One 13. https://doi.org/10.1371/journal.pone.0195477

Cross, M.R., Brughelli, M., Samozino, P., Morin, J.-B., 2017a. Methods of Power-Force-Velocity Profiling During Sprint Running: A Narrative Review. Sport. Med. 47, 12551269. https://doi.org/10.1007/s40279-016-0653-3

Cross, M.R., Brughelli, M., Samozino, P., Brown, S.R., Morin, J.-B., 2017b. Optimal Loading for Maximizing Power During Sled-Resisted Sprinting. Int. J. Sports Physiol. Perform. 12, 1069-1077. https://doi.org/10.1123/ijspp.2016-0362

Cross, M.R., Brughelli, M., Brown, S.R., Samozino, P., Gill, N.D., Cronin, J.B., Morin, J.B., 2015. Mechanical properties of sprinting in elite rugby union and rugby league. Int. J. Sports Physiol. Perform. 10, 695-702. https://doi.org/10.1123/ijspp.2014-0151

di Prampero, P.E., Botter, A., Osgnach, C., 2015. The energy cost of sprint running and the role of metabolic power in setting top performances. Eur. J. Appl. Physiol. 115, 451-469. https://doi.org/10.1007/s00421-014-3086-4

di Prampero, P.E., 2005. Sprint running: a new energetic approach. J. Exp. Biol. 208, 2809-2816. https://doi.org/10.1242/jeb.01700

Furusawa, K., Hill, A. V., Parkinson, J.L., 1927. The Dynamics of "Sprint" Running. Proc. R. Soc. B Biol. Sci. 102, 29-42. https://doi.org/10.1098/rspb.1927.0035

Haugen, T.A., Breitschädel, F., Samozino, P., 2018. Power-Force-Velocity Profiling of Sprinting Athletes. J. Strength Cond. Res. 1. https://doi.org/10.1519/JSC.0000000000002890

Hopkins, W.G., 2000. Measures of reliability in sports medicine and science. Sports Med. 30, 1-15. https://doi.org/10.2165/000007256-200030050-00006

Jiménez-Reyes, P., Cross, M., Ross, A., Samozino, P., Brughelli, M., Gill, N., Morin, J.-B., 2018. Changes in mechanical properties of sprinting during repeated sprint in elite rugby sevens athletes. Eur. J. Sport Sci. 1-10. https://doi.org/10.1080/17461391.2018.1542032 
Jiménez-Reyes, P., Samozino, P., García-Ramos, A., Cuadrado-Peñafiel, V., Brughelli, M., Morin, J.-B., 2018. Relationship between vertical and horizontal force-velocitypower profiles in various sports and levels of practice. PeerJ 6, e5937. https://doi.org/10.7717/peerj.5937

Mendiguchia, J., Edouard, P., Samozino, P., Brughelli, M., Cross, M., Ross, A., Gill, N., Morin, J.-B., 2016. Field monitoring of sprinting power-force-velocity profile before, during and after hamstring injury: two case reports. J. Sports Sci. 34, 535-41. https://doi.org/10.1080/02640414.2015.1122207

Morin, J.-B., Brughelli, M., Brown, S.R., Cross, M.R., Samozino, P., 2018. Letter to the Editor regarding «Sprint mechanics return to competition follow-up after hamstring injury on a professional soccer player: A case study with an inertial sensor unit based methodological approach» by I. Setuain, P. Lecumberri, and M. Izquierdo. J. Biomech. 66, 198-199. https://doi.org/10.1016/j.jbiomech.2017.09.044

Morin, J.-B., Edouard, P., Samozino, P., 2011. Technical ability of force application as a determinant factor of sprint performance. Med. Sci. Sport. Exerc. $43,1680-1688$. https://doi.org/10.1249/MSS.0b013e318216ea37

Nagahara, R., Morin, J.-B., Koido, M., 2016. Impairment of Sprint Mechanical Properties in an Actual Soccer Match: A Pilot Study. Int. J. Sports Physiol. Perform. 11, 893898. https://doi.org/10.1123/ijspp.2015-0567

Nagahara, R., Mizutani, M., Matsuo, A., Kanehisa, H., Fukunaga, T., 2017a. Association of step width with accelerated sprinting performance and ground reaction force. Int J. Sports Med. 38, 534-540. https://doi.org/10.1055/s-0043-106191

Nagahara, R., Botter, A., Rejc, E., Koido, M., Shimizu, T., Samozino, P., Morin, J.-B., 2017b. Concurrent validity of GPS for deriving mechanical properties of sprint acceleration. Int. J. Sports Physiol. Perform. 12, 129-132. https://doi.org/10.1123/ijspp.2015-0566

Pantoja, P.D., Saez de Villarreal, E., Brisswalter, J., Peyré-Tartaruga, L.A., Morin, J.-B., 2016. Sprint Acceleration Mechanics in Masters Athletes. Med. Sci. Sport. Exerc. 48, 2469-2476. https://doi.org/10.1249/MSS.0000000000001039

Pantoja, P.D., Carvalho, A.R., Ribas, L.R., Peyré-Tartaruga, L.A., 2018. Effect of weighted sled towing on sprinting effectiveness, power and force-velocity relationship. PLoS One 13, e0204473. https://doi.org/10.1371/journal.pone.0204473

Rabita, G., Dorel, S., Slawinski, J., Saez-de-Villarreal, E., Couturier, A., Samozino, P., Morin, J.B., 2015. Sprint mechanics in world-class athletes: A new insight into the limits of human locomotion. Scand. J. Med. Sci. Sport. 25, 583-594. https://doi.org/10.1111/sms.12389

Romero-Franco, N., Jiménez-Reyes, P., Castaño-Zambudio, A., Capelo-Ramírez, F., Rodríguez-Juan, J.J., González-Hernández, J., Toscano-Bendala, F.J., Cuadrado-Peñafiel, V., Balsalobre-Fernández, C., 2017. Sprint performance and mechanical outputs computed with an iPhone app: Comparison with existing reference methods. Eur. J. Sport Sci. 17, 386-392. https://doi.org/10.1080/17461391.2016.1249031

Rossi, J., Slotala, R., Samozino, P., Morin, J.B., Edouard, P., 2017. Sprint acceleration mechanics changes from children to adolescent. Comput. Methods Biomech. Biomed. Engin. 20, 181-182. https://doi.org/10.1080/10255842.2017.1382922

Samozino, P., 2018. A Simple Method for Measuring Force, Velocity and Power Capabilities and Mechanical Effectiveness During Sprint Running, in: Biomechanics of Training and Testing. Springer International Publishing, pp. 237-267. https://doi.org/10.1007/978-3-319-05633-3 11

Samozino, P., Rabita, G., Dorel, S., Slawinski, J., Peyrot, N., Saez de Villarreal, E., Morin, J.-B., 2016. A simple method for measuring power, force, velocity properties, and mechanical effectiveness in sprint running. Scand. J. Med. Sci. Sports 26, 648-658. https://doi.org/10.1111/sms.12490

Simperingham, K.D., Cronin, J.B., Pearson, S.N., Ross, A., 2017. Reliability of horizontal force-velocity-power profiling during short sprint-running accelerations using radar technology. Sport. Biomech. 3141, 1-12. https://doi.org/10.1080/14763141.2017.1386707

Slawinski, J., Termoz, N., Rabita, G., Guilhem, G., Dorel, S., Morin, J.-B., Samozino, P., 2017. How 100-m event analyses improve our understanding of world-class men's and women's sprint performance. Scand. J. Med. Sci. Sports 27, 45-54. https://doi.org/10.1111/sms.12627

Volkov, N.I., Lapin, V.I., 1979. Analysis of the velocity curve in sprint running. Med. Sci. Sports 11, 332-7. 